Session 1547

\title{
Experiments in High Frequency Circuit Design
}

\author{
Earl F. Owen \\ Brigham Young University
}

\begin{abstract}
\end{abstract}
Circuit design at high frequency involves some unique and challenging problems. In the Electronics Engineering Technology program at BYU students complete an experiment in the design and test of transistor amplifiers at frequencies above $1 \mathrm{GHz}$. The objective of the experiment is to give students the background they need to be familiar with the problems and methods of high frequency circuits they will encounter in industry. Special computer-aided design and analysis programs have been developed to demonstrate concepts of high frequency design. This paper will detail some of the topics students need to understand in design and test of high frequency circuits, and describe the computer programs that are used to assist them. These programs are patterned after standard commercial design and analysis programs, but are simplified to be adaptable to the learning environment rather than the professional. The computer programs contain graphics and animation to assist with teaching and learning objectives, while also providing the computer-assisted circuit design and simulation necessary at high frequencies. Topics learned include definition of s parameters, circuit design using s parameters and the Smith chart, impedance matching for gain and stability using s parameter design techniques, and microstrip circuit design and layout. In the process students also learn about the network analyzer and how to use this instrument to measure s parameters and analyze circuit performance.

\section{Introduction}

In the Electronics Engineering Technology program at Brigham Young University students complete an experiment in amplifier design and test at frequencies of $1 \mathrm{GHz}$ and above. The experiment is designed to introduce them to the distinctive methods and problems of high frequency circuit design and operation. Prior to introducing the principles of high frequency circuit design, students must have first completed the appropriate prerequisites, including basic principles of RF circuits, impedance matching, and transmission line theory including the use of the Smith chart. The microwave amplifier experiment includes the design steps necessary to extend circuit design and analysis from RF into microwave frequency range.

Before beginning their amplifier design, students must learn about s parameters and the $s$ parameter equivalent circuit used in circuit design and analysis at high frequency. Once they understand these concepts, they can measure the s parameters for a high frequency transistor using an s parameter test set. This introduces them to the network analyzer and the role this instrument plays in microwave circuit design and analysis. The measured s parameters are used to

Proceedings of the 2001 American Society for Engineering Education Annual Conference \& Exposition Copyright ${ }^{\circ}$ 2001, American Society for Engineering Education 
complete gain and stability analyses of the transistor to be used for the amplifier design. Then the circuit design is completed on the Smith chart. With a successful design the students then construct the matching circuits using microstrip and surface mount techniques. Not all the student amplifiers meet the original design specifications, but the process usually accomplishes the course learning objectives, understanding the methods and problems of high frequency circuit design, analysis and test.

The concepts of s parameters and amplifier design using the s parameter equivalent circuit is somewhat difficult for most students to understand. To assist in teaching the principles a computer design and analysis program named SPAR was developed by the author. The program has two purposes. First, it is intended as a teaching aid, and contains graphics and animation to help visualize the concept of the s parameter equivalent circuit. Students use this program while learning the concepts necessary to complete the experiment. Second, it contains automated routines used to simplify the calculations necessary to complete an amplifier design. The SPAR program can be downloaded from the class web page at http://class.et.byu.edu/eet345/.

S parameter definition and measurement.

To understand the process of amplifier circuit design at microwave frequencies students need to first learn what s parameters are, how they are obtained for a device such as a transistor, and how they are used in circuit design. The SPAR program uses graphics and animation to define the $\mathrm{s}$ parameter equivalent circuit of a device such as a transistor, and to demonstrate how this equivalent circuit is used in amplifier design and analysis. The s parameter equivalent circuit, shown in Figure 1, is called a signal flow graph, and is an equivalent representation of how signals

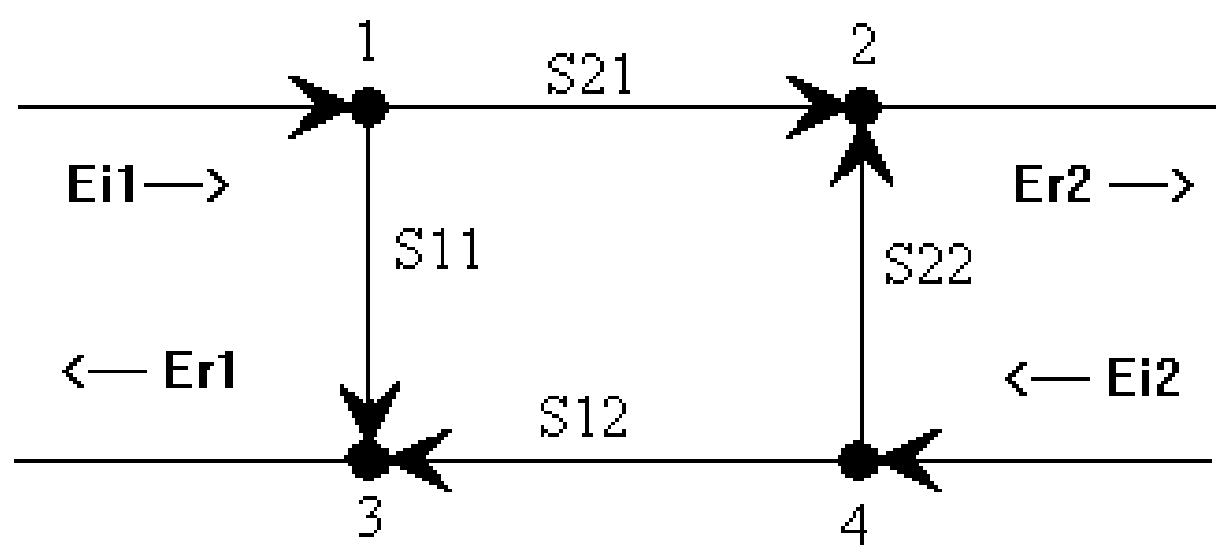

Figure 1. Signal flow graph equivalent of a transistor

Proceedings of the 2001 American Society for Engineering Education Annual Conference \& Exposition

Copyright $\odot$ 2001, American Society for Engineering Education 
flow in the actual transistor. An incident voltage $\mathrm{E}_{\mathrm{i} 1}$ at the input will divide, part will be reflected back through the $S_{11}$ path and the remainder transmitted to the output through the $S_{21}$ path.

Similarity a signal $E_{\mathrm{i} 2}$ incident at the output will divide, part will be reflected back through the $S_{22}$ path and the remainder transmitted to the input through the $S_{12}$ path. Assuming signals exist at both ports, the reflected voltages can be represented in terms of the incident voltages and the $s$ parameters using the following relationships:

$$
E_{r 1}=S_{11} E_{i 1}+S_{12} E_{i 2} \quad E_{r 2}=S_{21} E_{i 1}+S_{22} E_{i 2}
$$

The SPAR program defines the s parameters using these two equations.

The four s parameters represent the transmission and reflection coefficients of the transistor. $S_{11}$ is the input reflection coefficient and defines the input impedance. $S_{21}$ is the forward transmission coefficient and defines the transistor gain or amplification experienced by the signal as it passes through the transistor. $\mathrm{S}_{12}$ gives the amount of reverse gain, or feedback through the transistor. $\mathrm{S}_{22}$ is the output reflection coefficient, and defines the output impedance. Analysis consists of summing the signals at the input and output ports of the network, and defining circuit operation from the results. When measured s parameters are used in the equivalent circuit, it will predict actual transistor operation.

The signal flow graph equivalent circuit is easily adapted to a graphical representation, and the SPAR program demonstrates this by sending the signals through the equivalent circuit, as they do in the actual circuit. Seeing the operation of the s parameter equivalent circuit in animated form gives the students a clearer picture of how it represents the actual circuit. Then the process of measuring actual s parameters on an operating transistor has more meaning. The s parameter test set, or network analyzer, is merely an instrument that provides the signals for each branch and measures the corresponding transfer ratio or s parameter. The process of measuring the $\mathrm{s}$ parameters is not an absolutely necessary part of the experiment, it is the resulting s parameters that are needed to begin the circuit design process, and these could be obtained from data sheets. Making the actual measurements is an instructive process however, and provides the student with a better understanding of the parameters that will be used in the circuit design.

Microwave circuit design.

Once students have developed an understanding of s parameters, and have measured the parameters for their transistor, they are ready to begin the amplifier circuit design. Standard design procedures found in the literature are used ${ }^{1,2}$. The first step is plotting gain and stability circles on the Smith chart for the transistor being used, using the measured s parameters. This defines the possible amplifier options. Then an appropriate design can be selected from the resulting plots. The SPAR program does all the calculations and plots, while helping the designer to understand what they mean. An example of an amplifier being designed for $9 \mathrm{~dB}$ power gain is shown in Figure 2. The stability circles define the areas of the Smith chart that will provide stable operation. The gain circles define the areas of the Smith chart that will provide the desired

Proceedings of the 2001 American Society for Engineering Education Annual Conference \& Exposition Copyright $\odot$ 2001, American Society for Engineering Education 


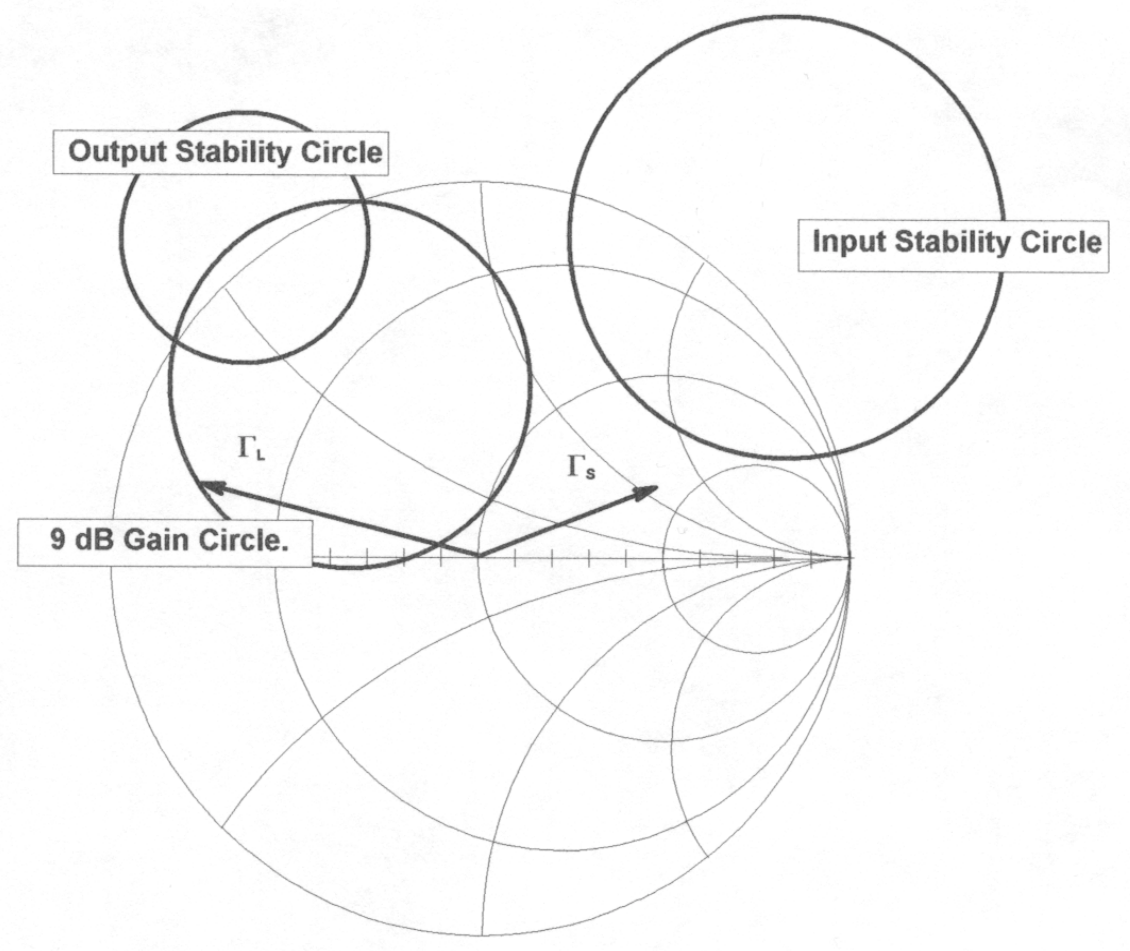

Figure 2. Amplifier design for a gain of $9 \mathrm{~dB}$.

amplifier gain. The vectors $\Gamma_{\mathrm{L}}$ and $\Gamma_{\mathrm{S}}$ give the impedance matching requirements for the amplifier, they must be chosen simultaneously in the correct areas of the Smith chart. This is accomplished with the aid of the SPAR program.

For a single stage amplifier a typical design consists of selecting matching circuits, the vectors of Figure 2, for the transistor input and output to provide the desired amplifier gain and stability. This is a complex process, since there are several options for a viable design. The input circuit has an effect on the transistor output, while the output circuit has an effect on the transistor input, so the design of each circuit must take into account the effects on the other. What this means is that you can't design the input circuit until you know the output circuit design, but you can't design the output circuit until you know the input circuit design, an apparent contradiction. The SPAR program allows the designer to view both circuit designs simultaneously, showing the effects of each circuit on the other, and on overall performance of the amplifier. In other words, both circuits are designed at the same time with the help of the SPAR program. In the example shown in Figure 2 the $\Gamma_{\mathrm{L}}$ (output) vector, which gives the output matching circuit, must be selected

Proceedings of the 2001 American Society for Engineering Education Annual Conference \& Exposition Copyright (C) 2001, American Society for Engineering Education 
somewhere on the $9 \mathrm{~dB}$ gain circle. As this vector is rotated to various points on the gain circle, the SPAR program calculates and displays the corresponding $\Gamma_{S}$ (input) vector.

Actual gain and stability data are shown in real time for the transistor that will be used in the amplifier. Three gain options are described in the literature, referred to as transducer gain $\mathrm{G}_{\mathrm{T}}$, power gain $\mathrm{G}_{\mathrm{P}}$, and available gain $\mathrm{G}_{\mathrm{A}}$. The SPAR program allows the designer to see the stability and gain circles on the Smith chart for each of these options, showing what they mean in the amplifier design. With the help of the circles plotted by the program, the designer selects which circuit to match and which to mis-match, corresponding to which gain option is to be used. Then each circuit can be optimized, while viewing its effects of the other, i.e., vary the design of the output circuit while watching the corresponding effects on the input. When a satisfactory design is completed, a circuit must be constructed corresponding to the design.

Constructing and testing the circuit

The amplifier circuit design procedure above gives the designer the reflection coefficients, or equivalently the impedances, required at the transistor input and output for the amplifier design. The designer must now select circuit components and a circuit layout that will provide these impedances. The SPAR program demonstrates several options for accomplishing this process. The designer studies these options to understand how they are constructed, then selects a design that can be accommodated on their board at their frequency. This may consist of circuits such as quarter wave impedance matches, open or shorted stub matches, or a combination of these with lumped elements such as capacitors. The matching circuits are completed in microstrip, using surface mount for any lumped circuit components such as resistors and capacitors.

Microstrip is the preferred circuit layout technique at frequencies in the GHz range, since it allows the design to be accomplished with transmission line sections. Using microstrip the designer can vary the line characteristic impedances and the line lengths as necessary to provide the correct impedances to the transistor. Impedance and wavelength on microstrip must be calculated using the complex mathematical relationships that describe propagation on this type of transmission line. The SPAR program completes these calculations, and provides the layout for the student to use for the final circuit arrangement. The circuit is then etched or milled into the board, and all surface mount components are soldered in. Then the amplifier is tested on the network analyzer, where the power gain is plotted as a function of frequency.

Figure 3 shows a picture of a completed student amplifier. The transistor is located at the center of the circuit, with the microstrip matching circuits placed between to the transistor and board input and output connections. These microstrip circuits provide the impedances designed earlier, shown by the input and output vectors on the Smith chart of Figure 2. The DC bias connection, along with the surface mount resistors and capacitors necessary for the dc bias circuit can also be seen on the circuit. The gain of this amplifier plotted as a function of frequency is shown in Figure 4. The amplifier has peak gain of almost $11 \mathrm{~dB}$ at $1.5 \mathrm{GHz}$, and $3 \mathrm{~dB}$ bandwidth of about 100 MHz. This was one of the better amplifiers, not necessarily typical. It is not easy for students to

Proceedings of the 2001 American Society for Engineering Education Annual Conference \& Exposition Copyright $(\odot)$ 2001, American Society for Engineering Education 


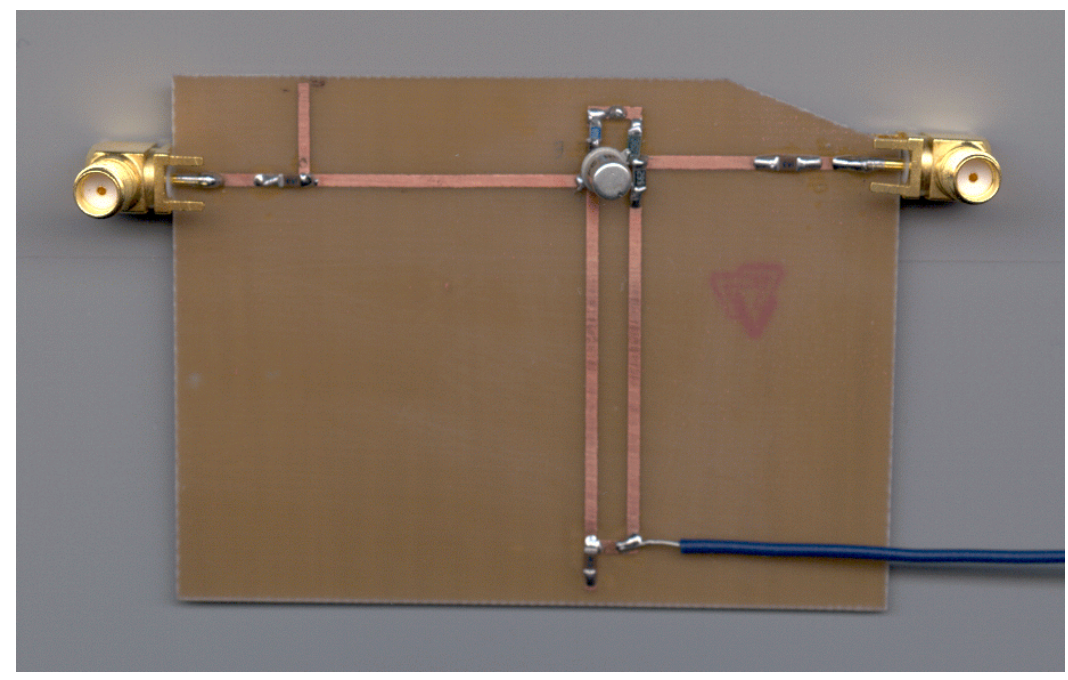

Figure 3. A student amplifier designed for $9 \mathrm{~dB}$ gain at $1.5 \mathrm{GHz}$.

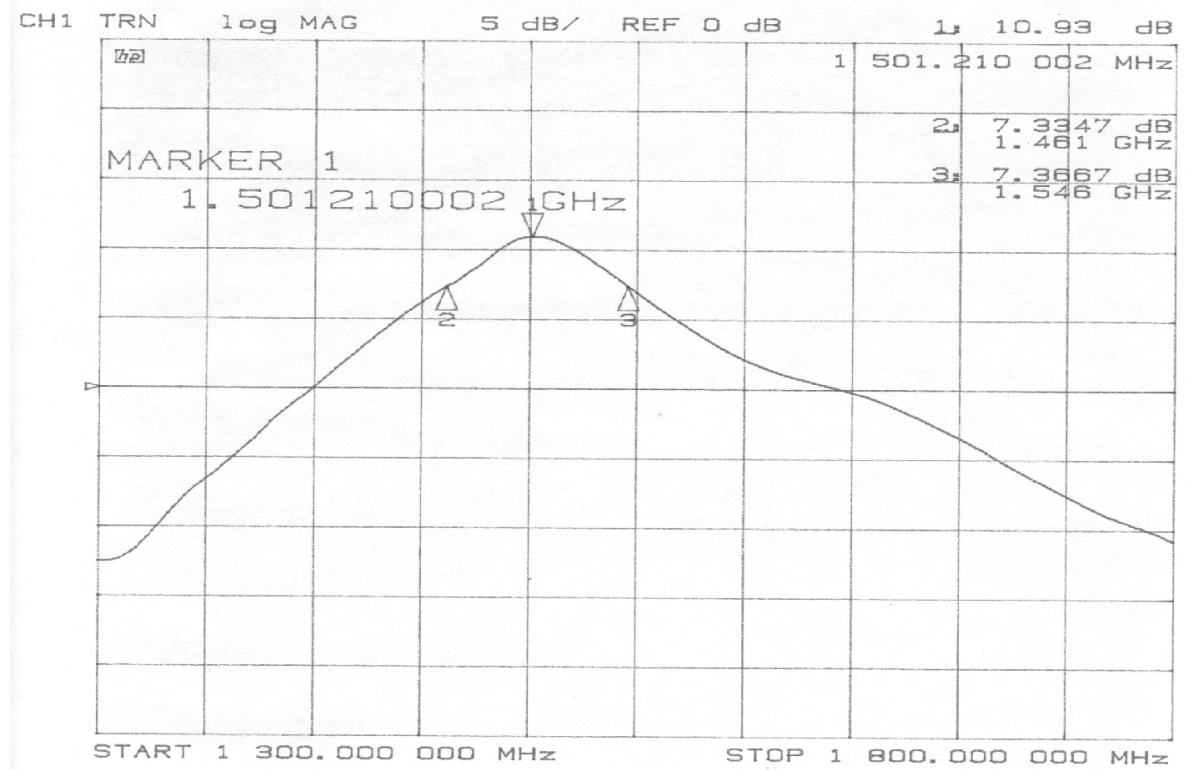

Figure 4. Gain vs. frequency response of the amplifier in Figure 3. 
get the performance shown in Figure 4 on the first try. Most student amplifiers have only 1 or 2 $\mathrm{dB}$ gain, some do not work at all.

\section{Student feedback}

In the most recent class of 21 students a survey was conducted to determine student feedback about the value of the high frequency amplifier experiment. Some of the results are as follows.

1. The objective of the s parameter lab is to familiarize you with some of the particular problems and characteristics of circuit design and operation at the high frequency and data rates being used in current analog and digital technology. How well did this lab accomplish this objective for you? Not at all (0\%) to Very Well $(100 \%)$.

Average response: $70 \%$.

2. How does the complexity of this lab compare with your expectations as an EET student? Much too easy (0\%) to Much too hard (100\%).

Average response: $60 \%$.

3.The lab was a valuable part of the curriculum. Disagree (0\%) to Agree (100\%). Average response: $72 \%$.

4. $60 \%$ of students stated they would still complete the experiment if the lab was available but not a part of the curriculum, just to learn the concepts. Of those who said they would not, the time involved was the main reason given.

5. $94 \%$ of students stated the lab was a meaningful experience.

\section{Conclusion}

The high frequency amplifier experiment described herein is used as an introduction to the techniques of high frequency circuit design. The experiment is intended to introduce students to the problems encountered in high frequency engineering, whether they are in analog of digital circuits. Students become aware of the effects of high frequency/high data rates in circuit operation. The SPAR program represents a major part of the lab, providing an introduction to the principles of microwave circuit design, and giving students the computer-aided design routines they need to complete the experiment. The concepts are learned by following the basic steps for completing a typical high frequency amplifier design:

1. Learn the definition of s parameters, and measure parameters for a high frequency transistor.

2. The SPAR program accepts the measured parameters, and gives the gain and stability information for the transistor, indicating how the transistor will perform in an amplifier circuit. 
3. Using the gain and stability information given in step 2, a circuit design is selected that will provide the desired amplifier operation.

4. The SPAR program provides options for achieving the desired circuit configuration, demonstrating the design concepts and doing the complex mathematical calculations. 5. The circuit is then constructed and tested with the help of the SPAR program and the network analyzer.

Some students who complete the amplifier lab plan to go on and develop further skills in RF and microwave engineering, eventually making this their professional area of interest. The experiment is a stepping stone in preparing them for further work in microwave circuit design. Many do not have this as their goal, but are more able to communicate with the microwave circuit design engineers they will interface within their careers. Students in both categories felt the high frequency amplifier experiment was a valuable experience.

References

1. "S_Parameter Design," Hewlett Packard Application Note 154, May 1973. This is one of the original and classic references for high frequency circuit design using s parameters.

2. Gonzalez, Guillermo, Microwave Transistor Amplifiers: Analysis and Design, $2^{\text {nd }}$ Ed. Prentice-Hall Inc., 1997.

\section{EARL F. OWEN}

Earl Owen has been teaching communications electronics courses in the Electronics Engineering Technology department in the School of Technology at Brigham Young University since 1982. Prior to his appointment at BYU he spent 10 years working in industry as an RF and microwave systems engineer, at Boeing Aerospace Co. in Seattle Washington and at TRW Inc. in Alice Springs, Australia. 